\title{
Haptic Feedback in Motor Hand Virtual Therapy Increases Precision and Generates Less Mental Workload
}

\author{
Cristina Ramírez-Fernández, Alberto L. Morán, Eloísa García-Canseco \\ Facultad de Ciencias \\ Universidad Autónoma de Baja California \\ Km. 103 Carretera Tijuana-Ensenada, 22860 Ensenada, México \\ Email: a302126,alberto.moran, eloisa.garcia@uabc.edu.mx
}

\begin{abstract}
In this work we show that haptic feedback in upper limb motor therapy improves performance and generates a lower mental workload. To demonstrate this, two groups of participants (healthy adults and elders with hand motor problems) used a low-cost haptic device (Novint Falcon) and a non-robotic device (Leap Motion Controller). Participants conducted the same rehabilitation task by using a non-immersive virtual environment. Results show significant differences for all participants regarding precision on the use of the haptic feedback device. Additionally, participants in the older adult group demonstrated a lower mental workload while using the haptic device (Novint Falcon). Finally, qualitative results show that participants preferred to conduct their therapy exercises by using the haptic device, as they found it more useful, easier to use and easier to learn.
\end{abstract}

Keywords-Haptic Feedback, Mental Workload, Motor Rehabilitation, Virtual Environment.

\section{INTRODUCTION}

Since more than 20 years ago specialists (i.e. therapists, physicians) incorporated the use of virtual environments (VEs) into upper limb motor rehabilitation [1]-[3]. This allowed to increase the intensity of the exercises, adapt repetitive tasks in real time, and obtain objective results from patients [4]. Depending on the state of the patient, VEs are used in hospitals, rehabilitation centers or patients' homes [5], and this under the supervision of a local or remote specialist [6].

To demonstrate the effectiveness of VEs in the upper limb motor therapy domain, several authors have evaluated their proposal with specialists and patients [7], [8]. These evaluations cover different perspectives, including usability, performance, and knowledge transfer from the virtual to the real environment, among others. From the usability perspective, specialists and patients use the VE to conduct a rehabilitation task and respond standard questionnaires to indicate their perception on usability factors (e.g. Technology Acceptance Model [9], System Usability Scale [10]) [11]. Additionally, from the performance perspective, the capability of the patient to conduct the exercises or tasks in the VE is evaluated, e.g. [12]-[14]. Finally, regarding knowledge transfer from the virtual to the real environment, it is determined by evaluating the patient after several therapy sessions by means of diverse clinical tests (e.g. Fulg-Meyer [15], Box and Block Test [16]) [17]. The clinical tests measure different aspects, for instance, the function and structure of the body and the activities of daily living, among others [18].

By executing therapy exercises, the patient interacts with an immersive or non-immersive VE, e.g., [13], [19], [20]. To do so, the patient uses robotic or non-robotic devices to conduct the exercises [21]-[24]. Preliminary evidence suggests that upper limb therapy using robotic devices is more effective than conducting it with non-robotic devices [25]-[29]. For instance, Cameirão et. al. [30] establishes that the results of the rehabilitation therapy of patients is closely related to the kind of device used.

However, demanding greater attention in the exercise can demand an excessive mental workload that may drive patients to abandon therapy. Kowalczewski and Prochazka [21], and Colombo et al. [31] establish therapy abandonment as one of the main problems in the motor rehabilitation process. For this reason, our main goal in this research is to determine the mental workload and performance of patients while they perform their motor hand therapy using a low cost robotic device (Novint Falcon haptic device [32]) and a (non-robotic) gesture sensor (Leap Motion Controller [33], [34]).

This paper is structured as follows. Section II presents related work on analysis of performance or mental workload in motor-hand virtual rehabilitation. Section III presents the methodology used to conduct our evaluation. The results of the evaluation in terms of performance and mental workload are presented in Section IV. Finally, Section V discusses our results and future research directions.

\section{RELATED WORK}

Performance and mental workload analysis allows to determine the effectiveness of a task by means of several devices or ways to provide feedback to a user. Weber et al. [35] conducted a task consisting in solving a cube puzzle in a VE with force, vibrotactile and visual feedback. The task was assigned to 30 students from a German aerospatial center, and consisted in moving and solving a cube from an initial to a target position. The results show that participants had a better 
performance when they were assisted with force feedback regarding precision, mental workload, and spatial orientation compared to vibrotactile and visual feedback systems. Collins et al. [36] analyses a haptic assisted training system for writing learning by using two haptic devices (Novint Falcon and Phantom Omni). Their results show that participants had a better performance (i.e. precision) in the trace of their letters when their are provided with haptic assistance. Furthermore, in their analysis they suggest the use of a Novint Falcon device in the initial training stage, in particular for students of special lectures; also they suggest that the Phantom Omni device could be used in more advanced training lessons using a more subtle haptic feedback. Cameirão et. al. [30] analyses the effect of a rehabilitation task based on a VE that uses three different interfaces: visual-based tracking, haptics, and a passive exoskeleton. In the experiment 44 chronic stroke patients participated, which were randomly assigned to a therapy task. Their results reveal significant improvements in almost all clinical scales used. Particularly, in the use of the VE with haptic feedback.

Our research determines the performance and mental workload of two groups of participants in a motor rehabilitation task by using a VE. To do so, 15 healthy older adults and 15 older adults with motor problems used two interaction devices. These devices were a low-cost robotic device and a hand gestural device.

\section{METHOD}

\section{A. System Set-up}

As a proof-of-concept of the design principles identified in our previous work [37], we developed the haptic maze VE. Haptic feedback is provided with the Novint Falcon haptic device and gesture feedback is provided with the Leap Motion controller. The Novint Falcon is a 3 degrees-of-freedom (dof) parallel robot that provides users with haptic feedback. It has a workspace of approximately $10.6 \mathrm{~cm}^{3}$. The Leap Motion controller is primarily designed for hand gesture and finger position detection in interactive software applications [34]. Open-source three-dimensional (3D) computer graphics software Blender [38] as well as the cross-platform game creation system Unity [39] were used to develop the VE. The user in the VE is represented by a proxy (or avatar) that moves in a 3D space. The collision detection algorithm is carried out with Unity libraries as well as with penalty-based methods [40], [41].

Our main objective in developing the haptic maze was to enhance the strength and wrist-movement accuracy of the patient. In the VE (Figure 1) we distinguish three main components: a) a configuration screen where the occupational therapist selects not only the visual features of the VE, but also the number of task repetitions, the simulated weight of the haptic proxy and the interaction device; b) the virtual maze; and c) the results screen where patient performance is displayed.

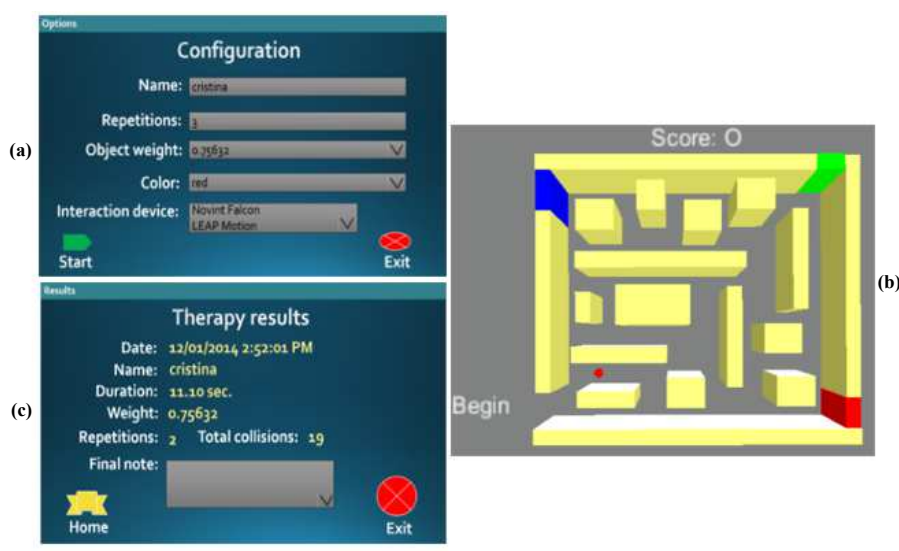

Fig. 1. Haptic-enhanced virtual environment: (a) therapy configuration; (b) exercise in the virtual maze for wrist therapy; (c) therapy results.

\section{B. Participants}

2 groups of 15 participants each were recruited. In the control group (G1), 15 healthy adults (8 women and 7 men, average age $55.73 \pm 7.56$ years) who live an independent life and have no apparent motor impairment problems served as participants. In the intervention group (G2), 15 elders ( 8 women and 7 men, average age $78.80 \pm 11.30$ years) with hand motor impairments, e.g., rheumatoid arthritis, were recruited from the elderly nursing homes "Casa Hogar del Anciano" and "Casa del Abuelo", both located in Ensenada, Baja California, Mexico. All participants were able to move their hand, only $50 \%$ of the healthy older adults declared to have experience on the use of the computer, and none of them declared to have experience on haptic devices or movement sensors.

\section{Experimental Design and Procedure}

The experiment followed a within subjects paradigm, i.e., both groups performed the task under the following conditions:

C1. Virtual environment with auditory, visual and haptic feedback using the Novint Falcon haptic device (top image in Figure 2).

C2. Virtual environment with auditory and visual feedback using the Leap Motion Controller (bottom image in Figure 2).

Participants of both groups performed one task with each interaction device consisting of moving an object through the maze to virtually "touch" three doors of different colors in the indicated order-red, green and blue-(Figure 3) with a simulated proxy weight of 100 gr (when using the haptic device). The interaction device was randomly assigned to participants when they performed their exercise. While moving the object through the labyrinth, if the participant collided the avatar with a wall, he was provided with auditory, visual and haptic feedback (see top image in Figure 2) or auditory and visual feedback (see bottom image in Figure 2).

After each exercise using any of these devices, each participant 


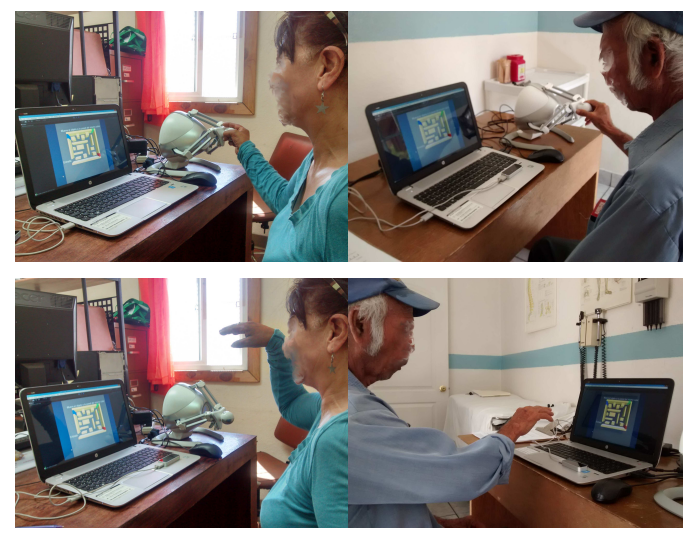

Fig. 2. Participants conducting the exercise with the Novint Falcon haptic device (above) and the Leap Motion device (below): adult with no motor impairment (left) and elder with motor impairment (right).

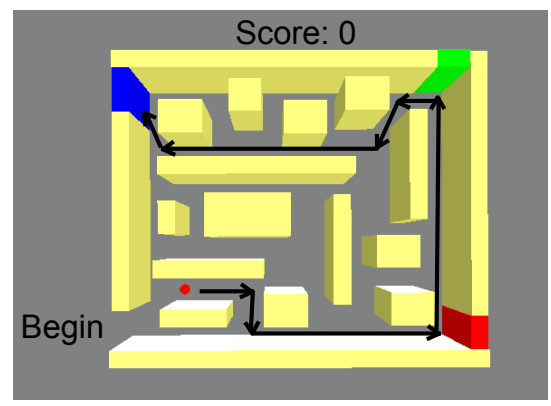

Fig. 3. Exercise in the haptic maze. The target trajectory motion is depicted in black. The proxy (red dot) changes its color to indicate the wall color that the user has to touch in the indicated order (red, green and blue).

filled out the NASA Task Load Index (TLX) questionnaire (20-point Likert scale) [42]. The NASA-TLX uses six dimensions to assess mental workload: mental demand, physical demand, temporal demand, performance, effort, and frustration. Table I shows the definitions of NASA-TLX dimensions. Lastly, after conducting the exercises with both devices participants were asked the following questions: a) Which device do you consider that allows you to have a more effective result in the therapy exercise? b) Which of the two devices was easier to use? c) Which of the two devices was easier to learn to use to conduct the task?.

We formulated the following hypotheses:

H1. Participants' performance (task execution and collisions detected) with auditory, visual and haptic feedback is higher than their performance with auditory and visual feedback.

H2. Participants' workload with auditory, visual and haptic feedback is lower than the workload with auditory and visual feedback

H3. G1 participants' performance (task execution and collisions detected) is different from that of G2 participants.

H4. G1 participants' workload is different from that of G2 participants.
TABLE I

RATING SCALE DEFINITIONS AND ENDPOINTS FROM THE NASA-TLX [42].

\begin{tabular}{lll}
\hline Title & Endpoints & Descriptions \\
\hline $\begin{array}{l}\text { Mental } \\
\text { Demand }\end{array}$ & Low/High & $\begin{array}{l}\text { How mentally demanding } \\
\text { was the task? }\end{array}$ \\
\hline $\begin{array}{lll}\text { Physical } \\
\text { Demand }\end{array}$ & Low/High & $\begin{array}{l}\text { How physically demanding } \\
\text { was the task? }\end{array}$ \\
$\begin{array}{lll}\text { Temporal } \\
\text { Demand }\end{array}$ & Low/High & $\begin{array}{l}\text { How hurried or rushed was } \\
\text { the pace of the task? }\end{array}$ \\
\hline Performance & Perfect/Failure & $\begin{array}{l}\text { How successful were you } \\
\text { in accomplishing what you } \\
\text { were asked to do? }\end{array}$ \\
& & $\begin{array}{l}\text { How hard did you have to } \\
\text { work to accomplish your } \\
\text { level of performance? }\end{array}$ \\
\hline $\begin{array}{l}\text { Erfort } \\
\text { Level }\end{array}$ & Low/High & $\begin{array}{l}\text { How insecure, discouraged, } \\
\text { irritated, stressed, and an- } \\
\text { noyed were you? }\end{array}$ \\
\hline
\end{tabular}

\section{RESULTS}

\section{A. Performance data}

Efficiency and Precision. The exercises were evaluated using objective performance data from the virtual environment, namely, the task execution time (efficiency) and the number of detected collisions with the virtual walls (precision of movement or accuracy). Table II presents a summary of the scores obtained by each participant. The scores were divided by time in seconds (efficiency) and number of collisions against the walls when the participant completed their exercise (precision), and this according to the interaction device used and participating group. We analysed the results of the therapy effectiveness in terms of performance of both groups (see Table III). As can be seen in Table III, we found no significant difference in task execution time (efficiency) between both devices, however we found significant difference on the precision of movement or accuracy. Additionally, Table IV shows the results of the analysis between groups and devices. Based on the significant differences found with the KruskalWallis test on the variance between groups, we later calculated the non-parametric Nemenyi test. Table IV, shows that there is a significant difference between G1 using the Novint Falcon haptic device and G2 using the Leap Motion controller on the precision of movement. In addition, we found significant difference in task execution time (efficiency) between G1 using the Novint Falcon haptic device and G2 using the Leap Motion controller.

\section{B. Subjective data}

Mental Workload. Table V shows a summary of the subjective mental workload results. As can be seen in Table III, we did not find a significant difference on mental workload between both devices for G1, but significant differences for G2. Analysing each of the mental workload sub scales for 
TABLE II

EVALUATION RESULTS OF THE EXERCISE IN TERMS OF PERFORMANCE (TASK EXECUTION TIME AND NUMBER OF COLLISIONS). G1: GROUP OF HEALTHY ADULTS, G2: GROUP OF ELDERLY WITH HAND MOTOR IMPAIRMENTS. SD: STANDARD DEVIATION.

\begin{tabular}{|c|c|c|c|c|c|c|c|c|}
\hline \multirow[t]{4}{*}{ Participant } & \multicolumn{8}{|c|}{ Outcome } \\
\hline & \multicolumn{4}{|c|}{ Time (sec) } & \multicolumn{4}{|c|}{ Number of collisions } \\
\hline & \multicolumn{2}{|c|}{ Novint Falcon } & \multicolumn{2}{|c|}{ Leap Motion } & \multicolumn{2}{|c|}{ Novint Falcon } & \multicolumn{2}{|c|}{ Leap Motion } \\
\hline & $\begin{array}{l}\text { G1 } \\
(\mathrm{SD}=\mathbf{2 4 . 4 6})\end{array}$ & $\begin{array}{l}\text { G2 } \\
(\mathrm{SD}=29.94)\end{array}$ & $\begin{array}{l}\text { G1 } \\
(\mathrm{SD}=31.70)\end{array}$ & $\begin{array}{l}\text { G2 } \\
(\mathrm{SD}=40.22)\end{array}$ & $\begin{array}{l}\text { G1 } \\
(\mathrm{SD}=16.18)\end{array}$ & $\begin{array}{l}\text { G2 } \\
(\mathrm{SD}=13.00)\end{array}$ & $\begin{array}{l}\text { G1 } \\
(\mathrm{SD}=35.24)\end{array}$ & $\begin{array}{l}\text { G2 } \\
(\mathrm{SD}=56.09)\end{array}$ \\
\hline 1 & 46.13 & 74.74 & 64.16 & 136.12 & 14 & 51 & 25 & 85 \\
\hline 2 & 51.35 & 109.74 & 46.67 & 162.30 & 15 & 21 & 20 & 129 \\
\hline 3 & 31.12 & 57.68 & 58.33 & 78.34 & 13 & 21 & 20 & 30 \\
\hline 4 & 43.87 & 84.48 & 60.49 & 73.17 & 19 & 36 & 51 & 65 \\
\hline 5 & 44.03 & 58.39 & 67.93 & 83.49 & 13 & 34 & 44 & 205 \\
\hline 6 & 94.37 & 79.57 & 118.76 & 77.18 & 19 & 17 & 55 & 36 \\
\hline 7 & 64.41 & 47.37 & 71.34 & 31.98 & 22 & 30 & 107 & 32 \\
\hline 8 & 26.94 & 27.26 & 118.21 & 123.47 & 18 & 13 & 92 & 54 \\
\hline 9 & 33.88 & 46.33 & 41.72 & 47.96 & 8 & 14 & 39 & 21 \\
\hline 10 & 69.34 & 89.51 & 76.74 & 129.35 & 18 & 33 & 82 & 55 \\
\hline 11 & 110.24 & 130.48 & 56.79 & 117.64 & 76 & 55 & 34 & 140 \\
\hline 12 & 35.32 & 52.13 & 142.24 & 30.81 & 8 & 25 & 141 & 52 \\
\hline 13 & 26.47 & 44.74 & 49.21 & 112.71 & 16 & 21 & 46 & 146 \\
\hline 14 & 42.32 & 84.56 & 60.60 & 98.93 & 13 & 26 & 43 & 141 \\
\hline 15 & 38.30 & 21.25 & 27.51 & 45.20 & 16 & 11 & 27 & 37 \\
\hline
\end{tabular}

G2 (see Table I) with a critical value: $U_{.01(1), 15,15}=169$, we did not find significant differences on mental demand ( $U=152)$, performance ( $U=63)$, and frustration level $(U=158.5)$. However, we found significant differences on G2 on physical demand $\left(U^{\prime}=174.5\right)$, temporal demand $\left(U^{\prime}=192\right)$, and effort $\left(U^{\prime}=187\right)$. Lastly, Table IV shows that we found significant differences on mental workload between both groups and devices for G1 using the Leap Motion controller and G2 using the Leap Motion controller, and G1 using the Novint Falcon haptic device and G2 using the Leap Motion controller.

Perceived usefulness, ease of use, and learnability. Figure 4 shows the results of questions to both groups (G1 and G2): a) Which device do you consider that allows you to have a more effective result in the therapy exercise? b) Which of the two devices was easier to use? c) Which of the two devices was easier to learn to use to conduct the task?. As can be seen in Figure 4, $80 \%$ of the participants of G1 and $86.7 \%$ of G2 considered that the Novint Falcon haptic device had greater usefulness (a). Additionally, Figure 4 shows that regarding the perception of ease of use (b), $86.7 \%$ of participants from G1 y $80 \%$ of participants from G2 considered the Novint Falcon haptic device as being easier to use. Lastly, regarding the perception on ease of learning (c), $86.7 \%$ of the participants from G1 and $80 \%$ of those from G2 considered the Novint
Falcon haptic device as being easier to learn (Figure 4).

Following conversations with participants, two additional topics have emerged about the usefulness, ease of use and learnability of devices, namely, task control and task support, which we explain in the next paragraphs.

Task Control. An interesting theme that emerged from the study was that participants considered that they had greater control to conduct the task using the haptic device. They said that they were able to synchronize their movement in the task

TABLE III

SUMMARY OF MANN-Whitney U TEST [43] ON H1 AND H2 HYPOTHESES (CRITICAL VALUE $U_{\alpha(1), 15,15}=169$ ) BETWEEN DEVICES. G1: GROUP OF HEALTHY ADULTS, G2: GROUP OF ELDERLY WITH HAND MOTOR IMPAIRMENTS.

\begin{tabular}{lcr}
\hline Group & Statistical value & Significance of difference \\
\hline & H1 (precision, $\alpha=0.01)$ & Significant \\
G1 & 213.5 & Significant \\
G2 & 201 & Not significant \\
\hline & H1 (efficiency, $\alpha=0.01)$ \\
G1 & 166 & Not significant \\
G2 & 147 & Not significant \\
\hline & H2 (mental workload, $\alpha=0.01)$ \\
G1 & 135 & Significant \\
G2 & 189.5 &
\end{tabular}




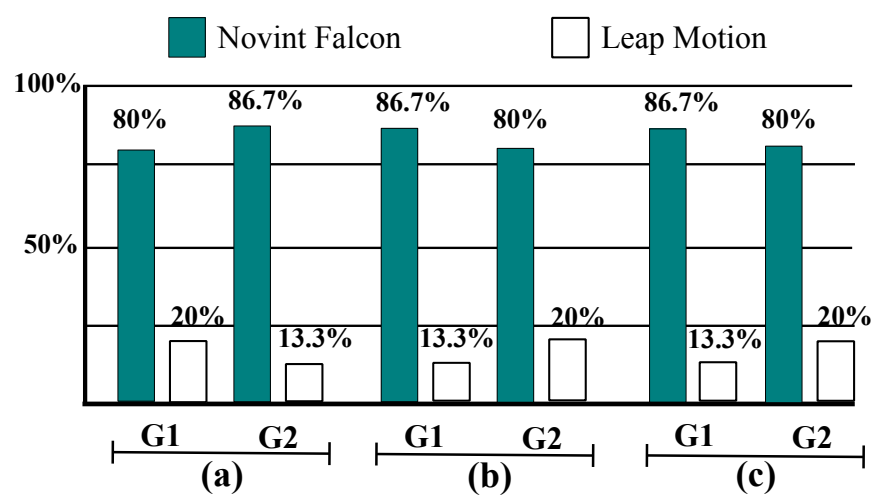

Fig. 4. Results of questions to both groups (G1 and G2): a) Which device do you consider that allows you to have a more effective result in the therapy exercise? b) Which of the two devices was easier to use? c) Which of the two devices was easier to learn to use to conduct the task?

in real time. Thus, statistically they were more precise, and participants from G2 perceived less mental workload (Table III). Some of the comments of members of G1 were: "I think that the device actually did what I wanted to do, it is more useful" (P6), "with the Novint device the difficulty of the task can be changing gradually, thus it is more challenging"( $\mathrm{P} 8)$, "the Novint is more useful because it actually obeys you, the other does not [obeys you]"(P14). In G2, some of the comments were: "the Novint is more useful because when it hits [the wall] you feel it"(P4), "to me this was something really new, it is as I could feel that I can touch something”(P9).

Task Support. Another interesting theme was that participants said that they felt being supported during the task while using the haptic device. This, because during their movements they felt the collitions of the proxy (their representation in the

TABLE IV

SUMMARY OF NEMENYI TEST [43] ON H3 AND H4 HYPOTHESES (CRITICAL VALUE $q_{\alpha, \infty, 4}=3.98$ ) BETWEEN GROUPS AND DEVICES. G1 GROUP OF HEALTHY ADULTS, G2: GROUP OF ELDERLY WITH HAND MOTOR IMPAIRMENTS.

\begin{tabular}{lcr}
\hline Comparative & $\begin{array}{l}\text { Statistical } \\
\text { value }\end{array}$ & $\begin{array}{l}\text { Significance } \\
\text { of } \\
\text { difference }\end{array}$ \\
\hline H3 (precision, $\alpha=0.025)$ & 1.34 & Not significant \\
G1 with Leap vs G2 with Leap & 3.31 & Not significant \\
G1 with Leap vs G2 with Novint & 7.14 & Significant \\
G1 with Novint vs G2 with Leap & 2.49 & Not significant \\
G1 with Novint vs G2 with Novint & 1.65 & Not significant \\
\hline H3 (efficiency, $\alpha=0.025)$ & 0.28 & Not significant \\
G1 with Leap vs G2 with Leap & 4.47 & Significant \\
G1 with Leap vs G2 with Novint & 2.54 & Not significant \\
G1 with Novint vs G2 with Leap & 5.11 & Significant \\
G1 with Novint vs G2 with Novint & 1.10 & Not significant \\
\hline \multicolumn{3}{c}{ H4 (mental workload, $\alpha=0.025)$} \\
G1 with Leap vs G2 with Leap & 6.17 & Significant \\
G1 with Leap vs G2 with Novint & 2.17 & Not significant \\
G1 with Novint vs G2 with Leap & \multicolumn{3}{c}{} \\
G1 with Novint vs G2 with Novint
\end{tabular}

TABLE V

EVALUATION RESULTS OF THE EXERCISE IN TERMS OF WORKLOAD (MENTAL, PHYSICAL AND TEMPORAL DEMAND, EFFORT, FRUSTRATION LEVEL AND PERFORMANCE). G1: GROUP OF HEALTHY ADULTS, G2: GROUP OF ELDERLY WITH HAND MOTOR IMPAIRMENTS. M:MEDIAN, IQR:INTER-QUARTILE RANGE.

\begin{tabular}{|c|c|c|c|c|}
\hline \multirow[t]{3}{*}{ Participant } & \multicolumn{4}{|c|}{ Outcome } \\
\hline & \multicolumn{2}{|c|}{ Novint Falcon } & \multicolumn{2}{|c|}{ Leap Motion } \\
\hline & $\begin{array}{l}\text { G1 } \\
M=29 \\
\text { IQR=21 }\end{array}$ & $\begin{array}{l}\text { G2 } \\
M=43.33 \\
I Q R=10.5\end{array}$ & $\begin{array}{l}\text { G1 } \\
M=36.66 \\
I Q R=15.16\end{array}$ & $\begin{array}{l}\text { G2 } \\
M=58 \\
I Q R=19.5\end{array}$ \\
\hline 1 & 23.33 & 86.33 & 35 & 54.66 \\
\hline 2 & 15 & 46 & 16 & 65.33 \\
\hline 3 & 45.33 & 61.66 & 31 & 70 \\
\hline 4 & 25.33 & 43.33 & 48 & 70.33 \\
\hline 5 & 31.66 & 62 & 36.66 & 50.66 \\
\hline 6 & 29 & 41.66 & 62 & 62.66 \\
\hline 7 & 21 & 43.33 & 16 & 46.33 \\
\hline 8 & 24.33 & 31 & 30.66 & 48.66 \\
\hline 9 & 23 & 34 & 24 & 41.33 \\
\hline 10 & 24.66 & 36 & 46 & 81.66 \\
\hline 11 & 53 & 38.33 & 36 & 68.33 \\
\hline 12 & 71.33 & 43.33 & 68.33 & 55 \\
\hline 13 & 44.33 & 31.66 & 39.33 & 73 \\
\hline 14 & 31.33 & 45 & 46 & 58 \\
\hline 15 & 50.33 & 29.33 & 38.33 & 47.33 \\
\hline
\end{tabular}

VE) and the walls of the maze. This perception of support was reflected in the significant differences in the precision of the task (see Table III). Some of the comments of participants from G1 were: "it is easier with the Novint Falcon device because you can feel the movement as you go"(P3), "I feel as it is easier [with the Novint] and I feel more secure while performing the exercise"(P5). Some of the comments from G2 were: "it is easier to move the hand with the Novint Falcon device, than having to hold your hand in the air"(P5), "it was easier [the Novint Falcon device] because there is not much problem due to having to hold your arm raised"(P6), "it is simpler [with the Novint Falcon device] because there is something to lean your arm to"(P9), "it is easier [with the Novint Falcon device] because there is something to hold your arm to"(P10).

\section{DISCUSSION AND CONCLUSION}

Participants' performance results from this study agree with those in Batchmann et al. [34], where it is established that an effective and efficient human-computer interaction (HCI) is strongly influenced by input devices. Our findings determined that the precision of the participants was significantly better, which may be due to the ease of delimiting the exercise with the Novint Falcon haptic device [44], [45]. However, no 
significant differences were found in the time of completion of the exercise. This behavior was reflected in the analysis between groups and devices that determined no difference in performance, except when G1 used the Novint Falcon haptic device and G2 used the Leap Motion controller.

Regarding the mental workload (NASA-TLX), the results establish that for participants of G1 the effort required to use any of the devices was about the same. However, for participants of G2 (seniors with motor impairments) using the Leap Motion device caused them a greater mental workload. During the execution of the exercise, one third of the participants of G2 (5 out of 15) had to hold their own hand to complete the task with the Leap Motion device. The above is confirmed by finding significant differences in the sub scales for physical demand, temporal demand, and effort. In addition, differences in workload between groups were found when using the Leap Motion controller, and in G1 while using the Novint Falcon haptic device and G2 while using the Leap Motion controller. This suggest that mental workload was higher in G2 given their motor impairment.

Moreover, in the concluding remarks of the evaluation several participants in both groups felt that the Leap Motion device did not correctly reflect the movement they had performed. This coincides with what has been reported by Guna and colleagues [33]. Guna et al. [33] establish that "the results of the dynamic scenario revealed the inconsistent performance of the controller, with a significant drop in accuracy for samples taken more than $250 \mathrm{~mm}$ above the controller's surface. The Leap Motion Controller undoubtedly represents a revolutionary input device for gesture-based human-computer interaction; however, due to its rather limited sensory space and inconsistent sampling frequency, in its current configuration it cannot currently be used as a professional tracking system".

Furthermore, our results show that the demographic characteristics and motor skills of the participants did not influence their perception of usefulness, ease of use and ease of learning of the interaction devices. Both groups considered that the Novint Falcon haptic device is more useful, easier to use and easier to learn to conduct the upper limb motor therapy exercise than the non-robotic device.

Concerning limitations, we would like to highlight what we consider the two main ones: the first limitation has to do with having conducted only one therapy session per participant; and thus, it is not possible to know which of the two devices and kinds of feedback generates better results on the long term (e.g. transference from the virtual to the real environment). The second limitation has to do with not having included actual upper limb motor therapy patients to compare their performance and mental workload. Thus, the scope of our results cannot necessarily be generalised to patients actually active in rehabilitation (e.g. hemiparesis).

For this reason, as future work we consider to address these limitations, by including patients that are actively conducting rehabilitation tasks, and for a longer time, so that we could have the opportunity to confirm our results at a longer term.

\section{ACKNOWLEDGMENT}

The authors would like to thank the elderly from "Casa Hogar de Anciano" and "Casa del Abuelo" in Ensenada, Baja California, México, for their valuable support and participation in this research work.

\section{REFERENCES}

[1] A. Bardorfer, M. Munih, A. Zupan, and A. Primozic, "Upper Limb Motion Analysis Using Haptic Interface," IEEE/ASME Transactions on Mechatronics, vol. 6, no. 3, pp. 253-260, 2001.

[2] V. G. Popescu, S. Member, G. C. Burdea, S. Member, M. Bouzit, and V. R. Hentz, "A Virtual-Reality-Based Telerehabilitation System with Force Feedback," IEEE Transactions on Information Technology in Biomedicine, vol. 4, no. 1, pp. 45-51, 2000.

[3] M. Holden and T. Dyar, "Virtual environment training-a new tool for neurorehabilitation," Neurology Report, vol. 26, no. 2, pp. 62-71, 2002

[4] M. Holden, "Virtual Environments for Motor Rehabilitation: Review," CyberPsychology \& Behavior, vol. 8, no. 3, pp. 187-211, 2005.

[5] M.-D. Chen and J. H. Rimmer, "Effects of exercise on quality of life in stroke survivors: a meta-analysis.," Stroke; a journal of cerebral circulation, vol. 42, pp. 832-7, Mar. 2011.

[6] M. Rogante, M. Grigioni, D. Cordella, and C. Giacomozzi, "Ten years of telerehabilitation: A literature overview of technologies and clinical applications," NeuroRehabilitation, vol. 27, pp. 287-304, 2010.

[7] E. M. J. Steultjens, J. Dekker, L. M. Bouter, J. C. M. van de Nes, E. H. C. Cup, and C. H. M. van den Ende, "Occupational therapy for stroke patients: a systematic review," Stroke, vol. 34, pp. 676-87, Mar. 2003.

[8] M. Zhang, T. C. Davies, and S. Xie, "Effectiveness of robot-assisted therapy on ankle rehabilitation-a systematic review.," Journal of neuroengineering and rehabilitation, vol. 10, p. 30, Jan. 2013.

[9] F. Davis, "Perceived Usefulness, Perceived Ease of Use, and User Acceptance of Information Technology," MIS Quarterly, vol. 13, no. 3, pp. 319-340, 1989.

[10] J. Brooke, "SUS - A quick and dirty usability scale," Usability evaluation in industry, pp. 189-194, 1996.

[11] P. Kortum and S. C. Peres, "The Relationship Between System Effectiveness and Subjective Usability Scores Using the System Usability Scale," International Journal of Human-Computer Interaction, vol. 30, pp. 575-584, May 2014.

[12] J. C. Stewart, S.-C. Yeh, Y. Jung, H. Yoon, M. Whitford, S.-Y. Chen, L. Li, M. McLaughlin, A. Rizzo, and C. J. Winstein, "Intervention to enhance skilled arm and hand movements after stroke: A feasibility study using a new virtual reality system.," Journal of neuroengineering and rehabilitation, vol. 4, p. 21, Jan. 2007.

[13] S. Subramanian, L. a. Knaut, C. Beaudoin, B. J. McFadyen, A. G. Feldman, and M. F. Levin, "Virtual reality environments for post-stroke arm rehabilitation," Journal of neuroengineering and rehabilitation, vol. 4, p. 20, Jan. 2007.

[14] H. C. Fischer, K. Stubblefield, T. Kline, X. Luo, R. V. Kenyon, and D. G. Kamper, "Hand rehabilitation following stroke: a pilot study of assisted finger extension training in a virtual environment.," Topics in stroke rehabilitation, vol. 14, no. 1, pp. 1-12, 2007.

[15] A. Fugl-Meyer, L. Jaasko, S. Leyman I. Olsson, and S. Steglind, "The poststroke hemiplegic patient. Part I. A method for evaluation of physical performance," Scand J. Rehab. Med., vol. 7, pp. 13-31, 1975.

[16] V. Mathiowetz, G. Volland, N. Kashman, and K. Weber, "Adult norms for the box and block test of manual dextery," The American Journal of Occupational Therapy, vol. 39, no. 6, pp. 386-391, 1985.

[17] F. D. Rose, E. A. Attree, B. M. Brooks, D. M. Parslow, and P. R. Penn, "Training in virtual environments: transfer to real world tasks and equivalence to real task training," Ergonomics, vol. 43, no. 4, pp. 494 $511,2000$.

[18] P. W. Duncan, "Outcome measures in stroke rehabilitation.," in Handbook of clinical neurology, vol. 110, pp. 105-11, Elsevier B.V., 1 ed., Jan. 2013.

[19] A. Henderson, N. Korner-Bitensky, and M. Levin, "Virtual reality in stroke rehabilitation: a systematic review of its effectiveness for upper limb motor recovery," Topics in stroke rehabilitation, vol. 14, no. 2, pp. 52-61, 2007. 
[20] A. Turolla, M. Dam, L. Ventura, P. Tonin, M. Agostini, C. Zucconi, P. Kiper, A. Cagnin, and L. Piron, "Virtual reality for the rehabilitation of the upper limb motor function after stroke: a prospective controlled trial," Journal of neuroengineering and rehabilitation, vol. 10, pp. 1-9, Jan. 2013.

[21] J. Kowalczewski and A. Prochazka, "Technology improves upper extremity rehabilitation.," in Progress in brain research, vol. 192, pp. 14759, Jan. 2011

[22] A. Rizzo and G. Kim, "A SWOT Analysis of the Field of Virtua Reality Rehabilitation and Therapy," Presence: Teleoperators and Virtual Environments, vol. 14, pp. 119-146, Apr. 2005.

[23] A. van Delden, C. Peper, G. Kwakkel, and P. Beek, "A systematic review of bilateral upper limb training devices for poststroke rehabilitation.," Stroke research and treatment, vol. 2012, p. 972069, Jan. 2012.

[24] P. Heo, G. M. Gu, S.-j. Lee, K. Rhee, and J. Kim, "Current hand exoskeleton technologies for rehabilitation and assistive engineering," International Journal of Precision Engineering and Manufacturing, vol. 13, pp. 807-824, May 2012.

[25] C. D. Takahashi, L. Der-Yeghiaian, V. Le, R. R. Motiwala, and C. S.C., "Robot-based hand motor therapy after stroke," Brain, vol. 131, pp. 425437, 2008.

[26] N. Norouzi-Gheidari, P. Archambault, and J. Fung, "Effects of robotassisted therapy on stroke rehabilitation in upper limbs: systematic review and meta-analysis of the literature.," Journal of rehabilitation research and development, vol. 49, pp. 479-96, Jan. 2012.

[27] I. Sarakoglou, S. Kousidou, N. G. Tsagarakis, and D. G. Caldwell, "Exoskeleton-Based Exercisers for the Disabilities of the Upper Arm and Hand," in Rehabilitation Robotics, no. August, pp. 499-522, 2007.

[28] D. J. Reinkensmeyer, J. L. Emken, and S. C. Cramer, "Robotics, motor learning, and neurologic recovery.," Annual review of biomedical engineering, vol. 6, pp. 497-525, Jan. 2004.

[29] S. Adamovich, G. Fluet, E. Tunik, and A. Merians, "Sensorimotor training in virtual reality: a review," NeuroRehabilitation, vol. 25 pp. 29-44, Jan. 2009.

[30] M. Cameirão, S. Badia, E. Duarte, A. Frisoli, and P. Verschure, "The combined impact of virtual reality neurorehabilitation and its interfaces on upper extremity functional recovery in patients with chronic stroke,' Stroke; a journal of cerebral circulation, vol. 43, pp. 2720-8, Oct. 2012.

[31] R. Colombo, F. Pisano, A. Mazzone, C. Delconte, S. Micera, M. C. Carrozza, P. Dario, and G. Minuco, "Design strategies to improve patient motivation during robot-aided rehabilitation.," Journal of neuroengineering and rehabilitation, vol. 4, pp. 1-12, Jan. 2007.

[32] Novint Falcon, http://www.novint.com

[33] J. Guna, G. Jakus, M. Pogačnik, S. Tomažič, and J. Sodnik, "An analysis of the precision and reliability of the leap motion sensor and its suitability for static and dynamic tracking.," Sensors, vol. 14, pp. 3702 20, Jan. 2014

[34] D. Bachmann, F. Weichert, and G. Rinkenauer, "Evaluation of the Leap Motion Controller as a New Contact-Free Pointing Device," Sensors, vol. 15 , pp. 214-233, 2015.
[35] B. Weber, M. Sagardia, T. Hulin, C. Preusche, and T. H. De, "Visual, Vibrotactile, and Force Feedback of Collisions in Virtual Environments: Effects on Performance, Mental Workload and Spatial Orientation," in VAMR/HCII 2013, vol. 1, pp. 241-250, 2013.

[36] M. Collins, W. Bulmer, S. Sharma, and J. Mayrose, "Haptics Assisted Training (HAT) System for children's handwriting," 2013 World Haptics Conference (WHC), pp. 559-564, Apr. 2013.

[37] C. Ramírez-Fernández, E. García-Canseco, and A. L. Morán, “Towards a Set of Design Principles for Hapto-Virtual Rehabilitation Environments: Preliminary Results in Fine Motor Hand Therapy," in Proceedings of the 8th International Conference on Pervasive Computing Technologies for Healthcare, vol. 5, pp. 394-397, ICST, 2014.

[38] Blender, http://www.blender.org

[39] Unity game engine, http://unity3d.com

[40] A. El Saddik, "Chapter 5: Computer Haptics," in Haptics Technologies, Springer Series on Touch and Haptic Systems, pp. 105-143, 2011.

[41] M. Moore and J. Wilhelms, "Collision Detection and Response for Computer Animation,” Computer Graphics, vol. 22, no. 4, pp. 289-298, 1988.

[42] S. Hart and L. Staveland, "Development of NASA-TLX (Task Load Index): Results of Empirical and Theorical Research," in Human Mental Workload, pp. 139-183, Elsevier, 1988.

[43] J. Zar, Biostatistical analysis. Prentice Hall, 2010.

[44] A. Basteris, S. Nijenhuis, A. Stienen, J. Buurke, G. Prange, and F. Amirabdollahian, "Training modalities in robot-mediated upper limb rehabilitation in stroke: a framework for classification based on a systematic review.," Journal of neuroengineering and rehabilitation, vol. 11, p. 111, Jan. 2014

[45] P. Maciejasz, J. Eschweiler, K. Gerlach-hahn, A. Jansen-troy, and S. Leonhardt, "A survey on robotic devices for upper limb rehabilitation," Journal of NeuroEngineering and Rehabilitation, pp. 1-29, 2014. 